\title{
A taxonomic study of Ooctonus (Hymenoptera, Mymaridae) from Heilongjiang, China
}

\author{
Hai-Feng Bai', Xiang-Xiang Jin', Cheng-De Li' \\ I School of Forestry, Northeast Forestry University, Harbin, 150040, China \\ Corresponding author:Xiang-Xiang Jin (jinxiangxiang006@gmail.com); Cheng-De Li (lichengde0608@sina.com)
}

Academic editor: M. Engel | Received 1 December 2014 | Accepted 18 January 2015 | Published 29 January 2015

http://zoobank.org/54946D24-18E8-4458-9175-26C0D826304F

Citation: Bai H-F, Jin X-X, Li C-D (2015) A taxonomic study of Ooctonus (Hymenoptera, Mymaridae) from Heilongjiang, China. ZooKeys 479: 25-36. doi: 10.3897/zookeys.479.9041

\begin{abstract}
Five species of Ooctonus Haliday (Hymenoptera, Mymaridae) from Heilongjiang Province, China, are reviewed. One species, $O$. huberi sp. n., is described as new, and four species, O. orientalis Doutt, O. saturn Triapitsyn, O. sublaevis Förster and O. vulgatus Haliday are reported as new to China. A key to the females of the 10 described Chinese species is given. All the specimens are deposited in the insect collections of Northeast Forestry University, China.
\end{abstract}

\section{Keywords}

Chalcidoidea, Mymaridae, Ooctonus, taxonomy, new species, China

\section{Introduction}

Ooctonus currently contains 36 described species: one in the Australian region (Perkins 1905), 12 in the Palaearctic region, five in the Oriental region (Triapitsyn 2010), 14 species in the Nearctic region including three also distributed in the Palaearctic region (Huber 2012), three in the Afrotropical region (Huber et al. 2010), and four in the Neotropical region (Huber 2013). Here we describe a new species, record 4 others for the first time from northeast China, and provide a key to females of the 10 Ooctonus species known from China. 


\section{Materials and methods}

Twenty-three specimens (19 females and 4 males) of Ooctonus were collected in Heilongjiang Province, northeast China by sweeping, Malaise traps (MT) or yellow pan traps (YPT). Specimens were dissected and mounted in Canada balsam on slides following the method described by Noyes (1982) and modified for Mymaridae by Huber (1988). Photographs were taken with a digital CCD camera attached to an Olympus BX51 compound microscope, and most measurements were made from slide-mounted specimens using an eye-piece reticle. Total body length excluding ovipositor was measured with an eye-piece reticle from alcohol-preserved specimens before being dissected. All measurements are given in micrometers $(\mu \mathrm{m})$. Triapitsyn (2010) and Huber (2012) should be consulted for depositories of type specimens, hosts, and literature references to species described from Palaearctic, Oriental, and Nearctic regions. Morphological terminology and abbreviations are those of Huber (2012). All the specimens listed below are deposited in Northeast Forestry University, Harbin, China (NEFU).

\section{Key to the females of Ooctonus species in China}

$1 \quad$ Metacoxa yellowish or brown, different in color from mesosoma .................. 2

- Metacoxa dark brown or black, almost same color as mesosoma ..................9

2 Frenum (Figs 27, 34) smooth medially, reticulate at lateral borders, sometimes also at anterior and posterior margins .................................................. 3

- $\quad$ Frenum (Figs $5,13,19)$ entirely reticulate, sometimes only faintly so.........4

3 Funicle with $2 \mathrm{mps}$ on $\mathrm{fl}_{5}$ and $\mathrm{fl}_{6}$; propodeum (Fig. 27) with median areole well separated from metascutellum by fairly long median carina, but the median carina often incomplete, not extending to anterior margin of propodeum, or almost absent

O. sublaevis

- $\quad$ Funicle without mps on $\mathrm{fl}_{5}$ and $\mathrm{fl}_{6}$; propodeum (Fig. 34) with median areole abutting metascutellum; the median carina absent and replaced by the two carinae forming inner margin of dorsolateral areoles

O. vulgatus Mesoscutum (Figs 13, 19) with median longitudinal groove, the groove sometimes very short at posterior margin or extending about $0.7 \times$ length of mesoscutum 5

Mesoscutum (Figs 4, 34) without median longitudinal groove .6 Funicle with 2 mps on $\mathrm{Al}_{5}$ and $\mathrm{f}_{6}$; plica (Fig. 13) bifurcate anteriorly with a long lateral and long medial arm....

O. orientalis Funicle without mps on $\mathrm{fl}_{5}$ and $\mathrm{fl}_{6}$; plica (Fig. 19) bifurcate anteriorly with a short lateral and short medial arm O. saturn Clava with 8 mps. O. insignis Haliday Clava with 7 mps.... O. notatus Walker Funicle without mps on $\mathrm{fl}$ Funicle with 1 or 2 mps on $\mathrm{fl}_{6}$ 
Propodeum with median areole separated from metascutellum by median carina; plica with an anterior bifurcation; mesosoma yellow; ovipositor relatively long, at least $1.1 \times$ as long as metatibia ....

O. novickyi Soyka

- $\quad$ Propodeum (Fig. 5) with median areole abutting metascutellum; the median carina absent and replaced by the two carinae forming inner margin of dorsolateral areoles; plica without an anterior bifurcation; mesosoma dark brown; ovipositor relatively short, at most $0.9 \times$ as long as metatibia

O. huberi Body length about $1300 \mu \mathrm{m}$; mesoscutum without median longitudinal groove or at most with very short one O. himalayus Subba Rao

- $\quad$ Body length about $2600 \mu \mathrm{m}$; mesoscutum with long median longitudinal groove (at least $0.5 \times$ length of mesoscutum)

O. sinensis Subba Rao

\section{Taxonomy}

\section{Ooctonus huberi Bai, Jin \& $\mathrm{Li}$, sp. $\mathrm{n}$.}

http://zoobank.org/9E177EE9-7AD7-4FB2-B8A1-57A71D8B23F4

Figs 1-11

Holotype. $q$ (NEFU) Harbin City, Maoershan Town, Mt. Maoershan, 700m. 18.VIII. 2014, Cheng-De Li, Hai-Feng Bai, Xiang-Xiang Jin, YPT.

Paratypes. 3 females, 2 males. Harbin City, Maoershan Town: Jianlagou. 4-17. VIII. 2014, Cheng-De Li, Hai-Feng Bai, Chao Zhang, Zhi-Guang Wu (2 o $q$, NEFU), MT; same data as holotype (2 ふ̋ ふૈ, NEFU); Laoyeling. 16-29.VIII. 2013, Cheng-De Li, Hai-Feng Bai (1 $q$, NEFU), MT.

Diagnosis. Funicle (Fig. 2) with 2 mps at least on $\mathrm{fl}_{4}-\mathrm{fl}_{8}$ and $7 \mathrm{mps}$ on clava; mesoscutum (Fig. 4) without median longitudinal groove; frenum (Figs 4, 5) entirely reticulate; propodeum (Fig. 5) with median areole abutting metascutellum; the median carina absent and replaced by the two carinae forming inner margin of dorsolateral areoles; plica without an anterior bifurcation; petiole 3.54-4.05x as long as wide; ovipositor (Fig. 7) slightly exserted, about $0.9 \times$ as long as gaster, and $0.86-0.90 \times$ as long as metatibia.

Ooctonus huberi sp. n. runs to O. novickyi in Triapitsyn's key (2010), and the differences are shown in the key above. The new species is also similar to O. lokomotiv. Both species have $1 \mathrm{mps}$ sometimes on $\mathrm{fl}_{3}$ and 2 mps on $\mathrm{fl}_{4}-\mathrm{fl}_{8}$ and have reticulation on mesoscutum and frenum, but $O$. huberi sp. n. differs from the latter by having $7 \mathrm{mps}$ on the clava ( $8 \mathrm{mps}$ in $O$. lokomotiv); petiole $3.54-4.05 \times$ as long as wide $(2.6-3.3 \times$ in $O$. lokomotiv); and ovipositor $0.86-0.90 \times$ as long as metatibia (1.2-1.4x in O. lokomotiv).

Description. Female. Body length 1240-1380. Head and mesosoma dark brown, metasoma brown; scape and pedicel mostly yellow except dorsally dark brown; $f_{1}$ brown, remainder of funicle dark brown; petiole and legs yellow except apical tarsomere brown.

Head. Head (Fig. 1) width 396-406. Vertex without stemmaticum. Mid ocellus diameter 29-31. Vertex with conspicuous reticulate sculpture; face with faint, inconspicuous reticulate sculpture. 


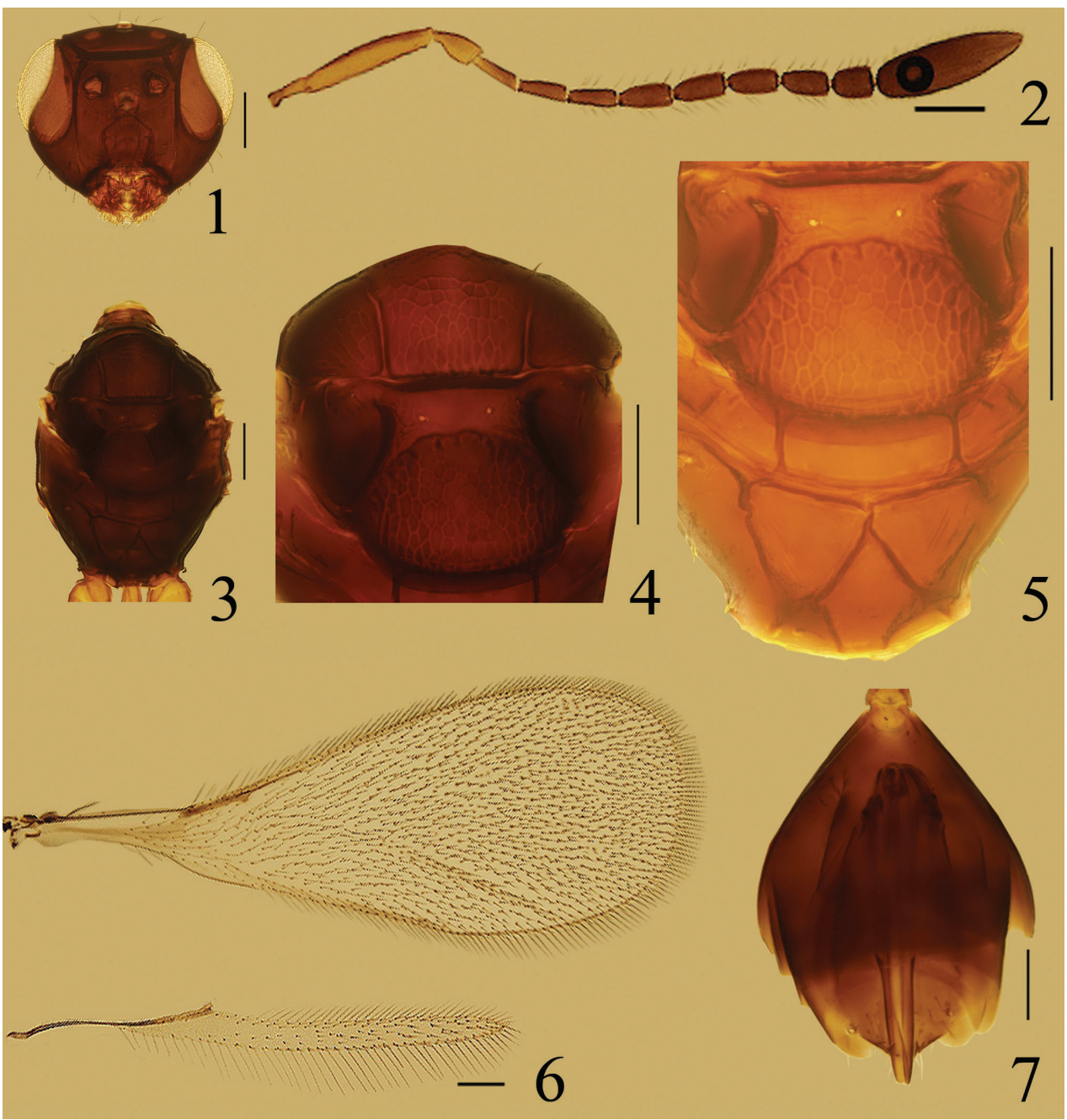

Figures I-7. Ooctonus huberi sp. n., holotype female (Jianlagou): I head, dorsal $\mathbf{2}$ antenna $\mathbf{3}$ mesosoma, dorsal $\mathbf{4}$ mesoscutum and scutellum, dorsal $\mathbf{5}$ frenum and propodeum, dorsal $\mathbf{6}$ wings $\mathbf{7}$ gaster, dorsal. Scale bars $=100 \mu \mathrm{m}$.

Antenna. Antenna (Fig. 2) with scape 4.61-5.25x as long as wide, slightly longitudinally striate; pedicel slightly longer than $\mathrm{f}_{1}$; funicle with $2 \mathrm{mps}$ on $\mathrm{f}_{4}-\mathrm{fl}_{8}$ and $7 \mathrm{mps}$ on clava, and sometimes $\mathrm{fl}_{3}$ with $1 \mathrm{mps}$ on one antenna. Clava 3.17-3.31 $\times$ as long as wide, slightly longer than $\mathrm{fl}_{6}-\mathrm{fl}_{8}$ together. Measurements (length/width): radicle 53, scape 199-204/ 38-43, pedicel 65-72/ 36-38, fl 60-72/ 22-24, fl 70-79/ 24-26, $\mathrm{fl}_{3} 72-77 / 26-29, \mathrm{fl}_{4} 77-82 / 29-34, \mathrm{f}_{5} 77-79 / 31-36, \mathrm{fl}_{6} 72-77 / 31-36, \mathrm{fl}_{7} 70-79 /$ $36-38, \mathrm{f}_{8} 58-65 / 43-46$, clava 221-240/ 70-74.

Mesosoma. Mesosoma (Fig. 3) with pronotum weakly sculptured. Mid lobe of mesoscutum (Fig. 4) with meshes raised; scutellar setae long, extending posterior to 


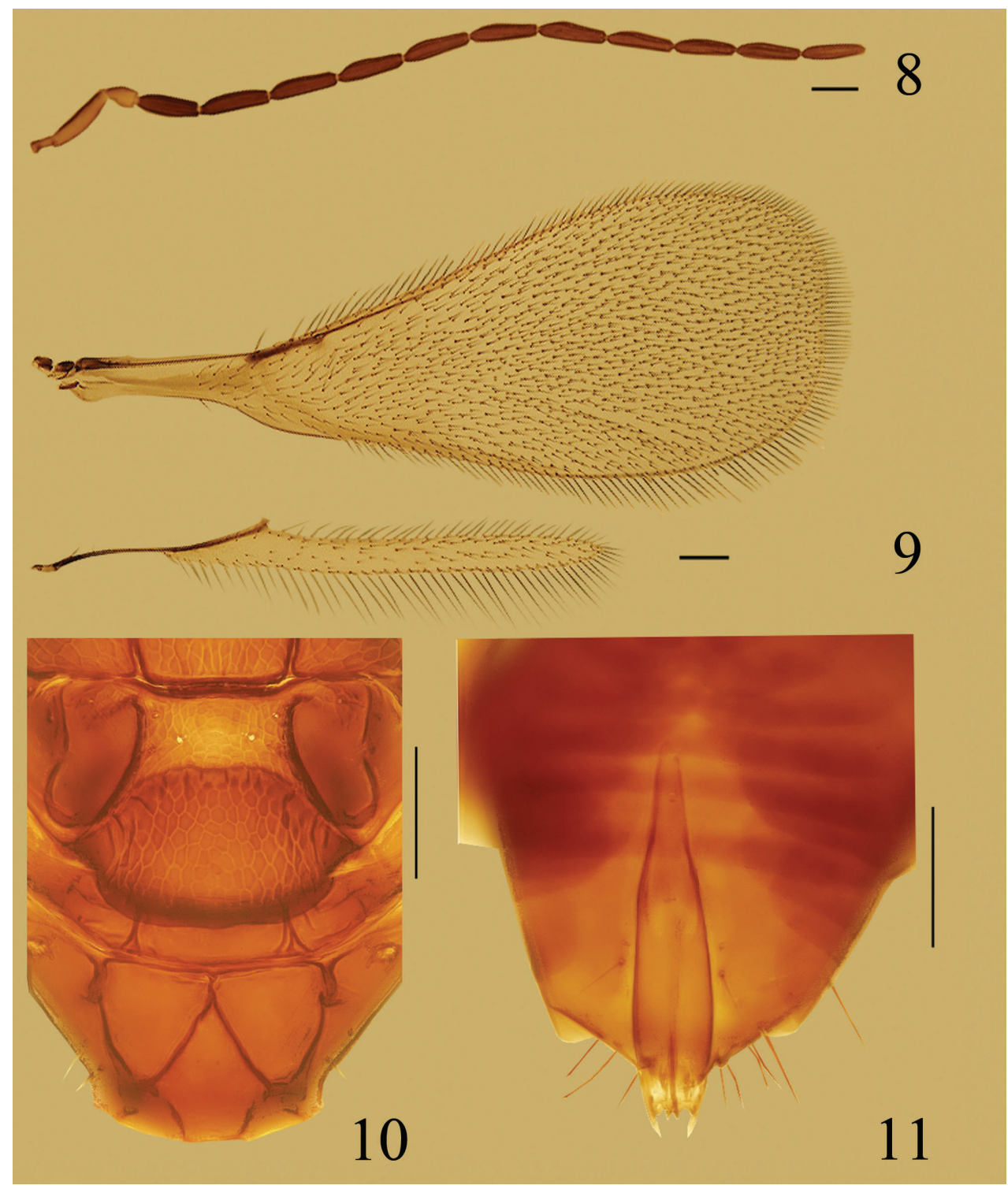

Figures 8-I I. Ooctonus huberi sp. n., paratype male (Jianlagou): 8 antenna $\mathbf{9}$ wings $\mathbf{1 0}$ posterior part of mesoscutum to propodeum, dorsal I I genitalia. Scale bars $=100 \mu \mathrm{m}$.

medially concave frenal line; frenum $0.69-0.75 \times$ mesoscutellum length and entirely reticulate. Metanotum with metascutellum smooth. Propodeum (Fig. 5) smooth between carinae and its anterior margin with a stub slightly lateral to lateral margin of metascutellum; median areole abutting metascutellum; the median carina absent and replaced by the two carinae forming inner margin of dorsolateral areoles; plica almost straight, extending almost to anterior margin of propodeum just medial to stub, without an anterior bifurcation but with a slight curved thickening posterior to the stub. 
Wings. Fore wing (Fig. 6) length 1415-1512, width 512-585, length/width 2.57-2.76, longest marginal setae $77-84,0.13-0.15 \times$ as long as greatest wing width. Marginal vein length 125-132. Hind wing (Fig. 6) length 1049-1122, width 67-70, length/width 16-17, longest marginal setae 122-125.

Metasoma. Petiole 3.54-4.05 $\times$ as long as wide, $1.35-1.38 \times$ as long as metacoxa, shorter than metacoxa + metatrochantellus. Gaster (Fig. 7) with ovipositor length 455-485, slightly exserted, 0.89-0.91× as long as gaster, and 0.86-0.90× as long as metatibia (515-525).

Male. Body length 1230-1310. Mid ocellus diameter 29-31. Antenna (Fig. 8). Measurements, length: radicle 48-50, scape 139-144, pedicel 60-70, $\mathrm{fl}_{1} 125, \mathrm{fl}_{2} 137-$ 144, $\mathrm{fl}_{3}$ 142-144, $\mathrm{f}_{4} 134-139, \mathrm{f}_{5} 142, \mathrm{fl}_{6} 137-142, \mathrm{fl}_{7}$ 137-142, $\mathrm{f}_{8} 134-139, \mathrm{fl}_{9}$ $130, \mathrm{fl}_{10} 132-137, \mathrm{fl}_{11} 134-142$. Total flagellar length $1537-1561 . \mathrm{Fl}_{6}$ length/width 4.21-4.38, with 7 mps. Fore wing (Fig. 9) length 1463-1512, width 561-585, length/ width 2.50-2.70, longest marginal setae $89-101,0.15-0.18 \times$ as long as greatest wing width. Hind wing (Fig. 9) length 1073-1122, width 72, length/width 14.91-15.58, longest marginal setae $132-134,1.83-1.87 \times$ as long as greatest wing width.

Host. Unknown.

Etymology. This species is named in honor of JT Huber, of the Canadian Forest Service, Ottawa, Canada.

\section{Ooctonus orientalis Doutt, 1961}

Figs $12-15$

Triapitsyn 2010: 36-40 (redescription, primary type data, distribution).

Specimens examined. 3 ㅇ q. Harbin City, Maoershan Town: Laoyeling. 10-11.VI. 2013, Xiang-Xiang Jin, Si-Zhu Liu, Chao Zhang, sweeping (1 ㅇ); Laoshan. 12-14.VI. 2013, Xiang-Xiang Jin, Si-Zhu Liu, Chao Zhang, YPT (1 + ); Jianlagou. 19.VII. 2014, Cheng-De Li, Hai-Feng Bai, Xiang-Xiang Jin, Yan Gao, YPT (1 ㅇ).

Diagnosis. Funicle (Fig. 12) usually with 2 mps on $\mathrm{f}_{5}-\mathrm{fl}_{8}$ and 7 mps on clava; mesoscutum (Fig. 13) with median longitudinal groove, the groove sometimes very short at posterior margin of mesoscutum or extending about $0.7 \times$ length of mesoscutum; frenum entirely reticulate; propodeum (Fig. 13) with median areole separated from metascutellum by long median carina; plica bifurcate anteriorly with a long lateral and shorter medial arm.

\section{Ooctonus saturn Triapitsyn, 2010}

Figs 16-24

Triapitsyn 2010: 36-40 (description, type data, distribution).

Specimens examined. 7 ㅇ ,, $2 \hat{\jmath}$. Harbin City, Maoershan Town: Jianlagou. 1-17.VI. 2014, Cheng-De Li, Hai-Feng Bai, Ye Chen, Chao Zhang, MT (3 ㅇ ㅇ); 


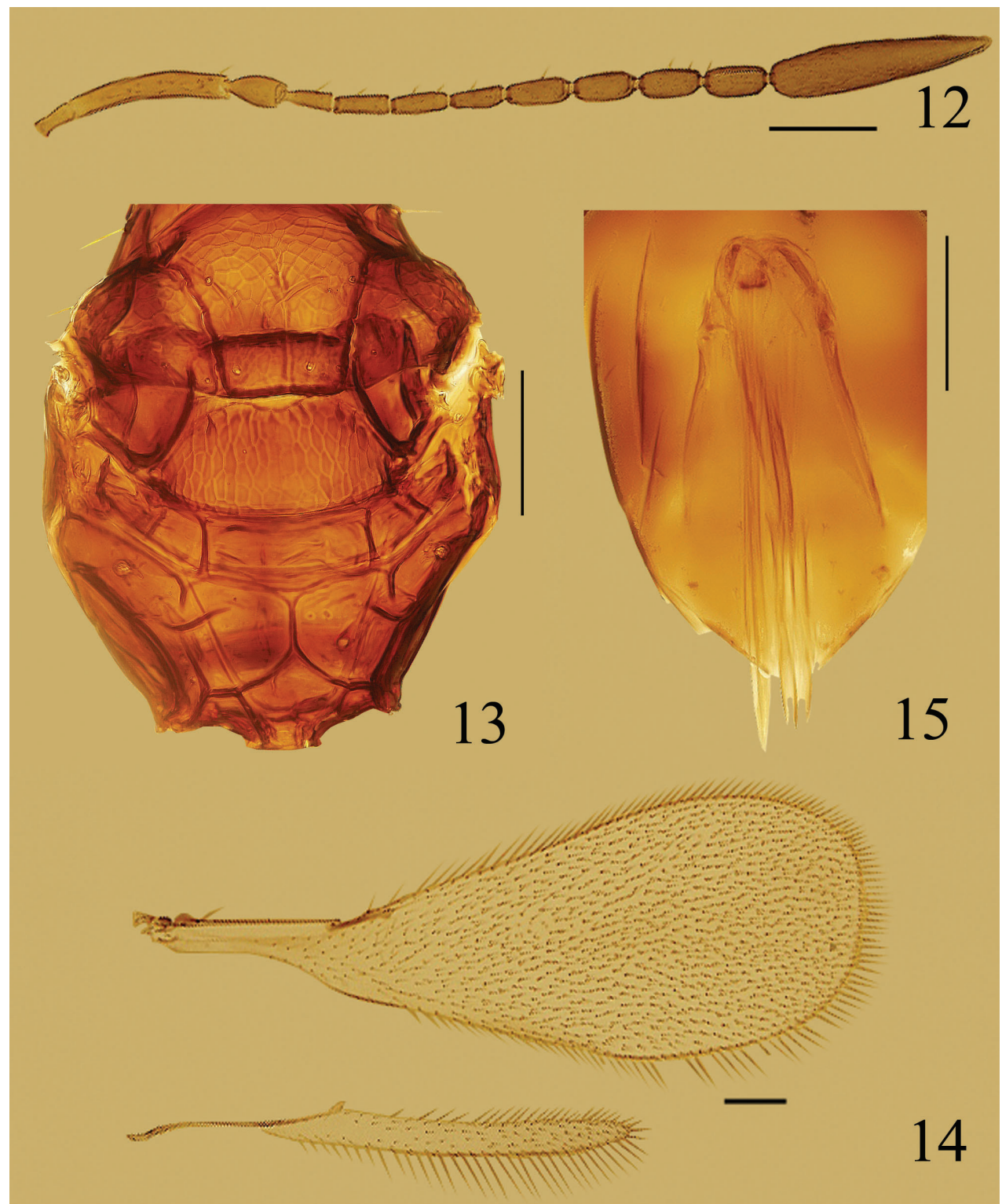

Figures 12-15. Ooctonus orientalis, female (Laoyeling): I 2 antenna 13 part of mesosoma, dorsal 14 wings $\mathbf{I} \mathbf{5}$ ovipositor. Scale bars $=100 \mu \mathrm{m}$.

Jianlagou. 4.VIII. 2014, Cheng-De Li, Hai-Feng Bai, Xiang-Xiang Jin, Yan Gao, sweeping (1 q); Laoshan. 12-14.VI. 2013, Xiang-Xiang Jin, Si-Zhu Liu, Chao Zhang, YPT (1 +); Jianlagou. 17.VI. 2014, Cheng-De Li, Hai-Feng Bai, Ye Chen, Chao Zhang, YPT $(2$ q, , 2 ふึ).

Diagnosis. Funicle (Fig. 16) with 2 mps on $\mathrm{fl}_{7}$ and $\mathrm{fl}_{8}$ and $7 \mathrm{mps}$ on clava; mesoscutum (Fig. 19) with median longitudinal groove, the groove sometimes very short 


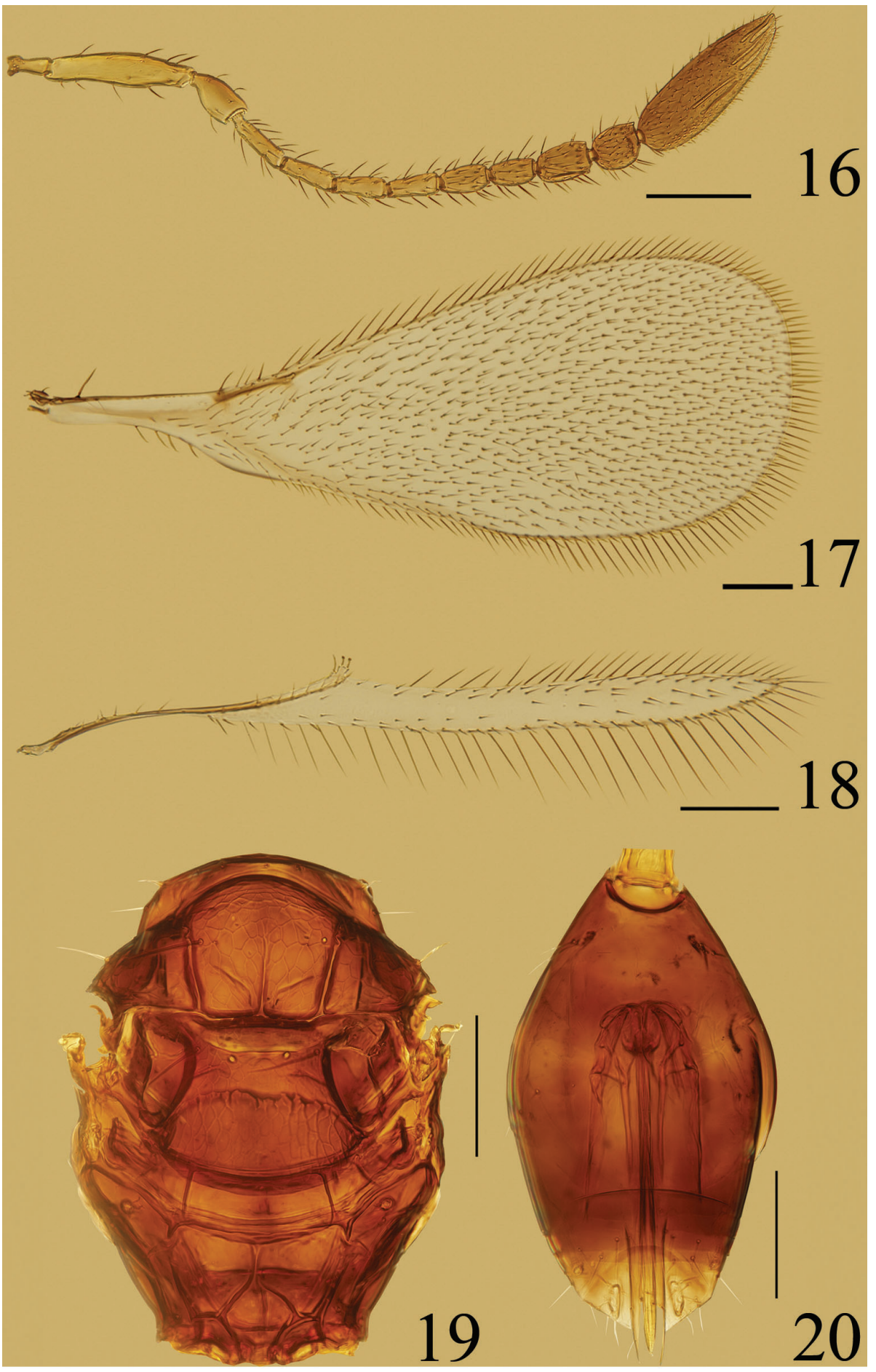

Figures 16-20. Ooctonus saturn, female (Jianlagou): $\mathbf{1 6}$ antenna $\mathbf{I 7}$ fore wing $\mathbf{1 8}$ hind wing $\mathbf{1 9}$ mesosoma, dorsal 20 gaster. Scale bars $=100 \mu \mathrm{m}$. 


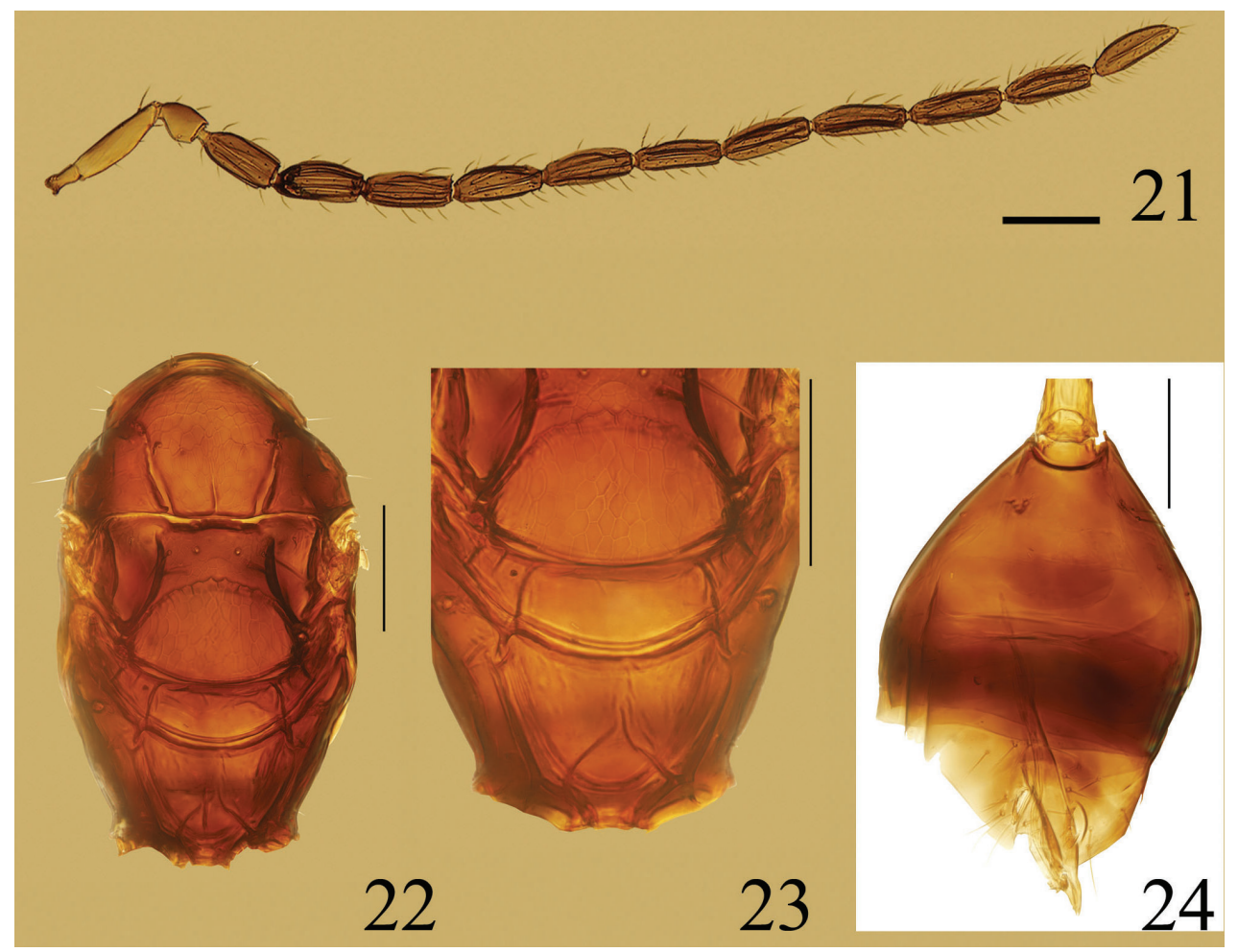

Figures 2I-24. Ooctonus saturn, male (Jianlagou): $2 \mathbf{I}$ antenna $\mathbf{2 2}$ mesosoma, dorsal $\mathbf{2 3}$ part of mesosoma, dorsal, 24 gaster. Scale bars $=100 \mu \mathrm{m}$.

at posterior margin of mesoscutum or extending about $0.5 \times$ length of mesoscutum; frenum entirely reticulate; propodeum (Fig. 15) with median areole separated from metascutellum by median carina; plica with a short bifurcation anteriorly.

\section{Ooctonus sublaevis Förster, 1847}

Figs 25-29

Specimens examined. 4 q 9 : Harbin City, Maoershan Town, Laoyeling. 10-11. VI. 2013, Xiang-Xiang Jin, Si-Zhu Liu, Chao Zhang, sweeping (1 o)); Yichun City, Wuying Town, Fenglin Natural Reserve. 3-4.VII. 2013, Guo-Hao Zu, Hui Geng, Si-Zhu Liu, Yang Peng, sweeping ( 3 우).

Diagnosis. Funicle (Fig. 25) usually with $2 \mathrm{mps}$ on $\mathrm{fl}_{5}-\mathrm{fl}_{8}$ (occasionally $\mathrm{fl}_{6}$ with just $1 \mathrm{mps}$ ) and $7 \mathrm{mps}$ on clava; mesoscutum (Fig. 27) usually without median longitudinal groove, rarely with a very short groove; frenum with weak reticulate sculpture; propodeum (Fig. 27) with median areole well separated from metascutellum by fairly long median carina, but the median carina often incomplete, not extending to anterior margin of propodeum, or almost absent; plica straight or slightly curved outward and not divided anterodorsally. 


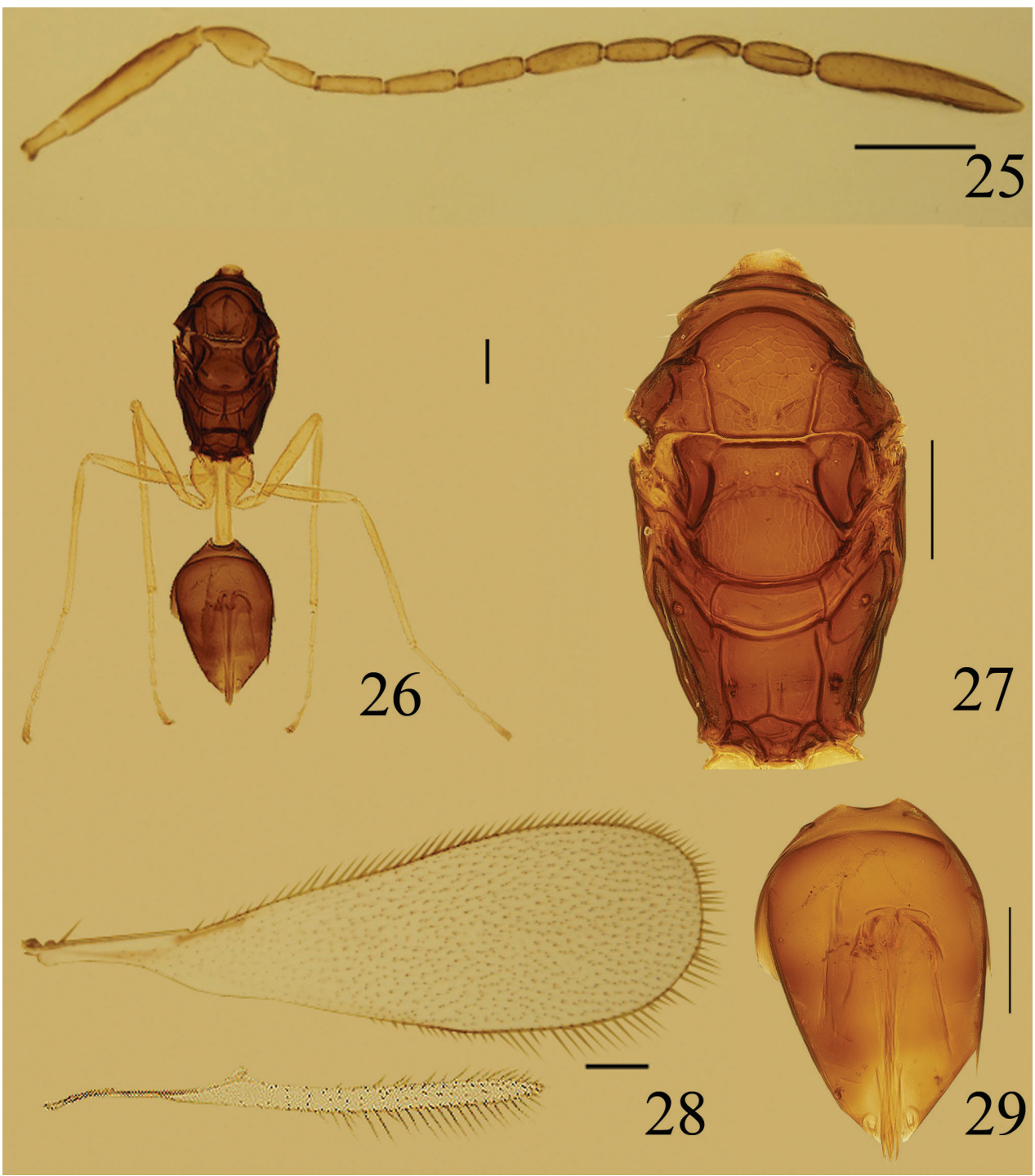

Figures 25-29. Ooctonus sublaevis, female (Fenglin Natural Reserve): $\mathbf{2 5}$ antenna $\mathbf{2 6}$ body, dorsal $\mathbf{2 7}$ mesosoma, dorsal $\mathbf{2 8}$ wings $\mathbf{2 9}$ gaster. Scale bars $=100 \mu \mathrm{m}$.

\section{Ooctonus vulgatus Haliday, 1833}

Figs 30-35

Specimen examined. 1 q. Harbin City, Maoershan Town, Laoyeling. 17.VI. 2014, Cheng-De Li, Hai-Feng Bai, Guo-Hao Zu, Ye Chen, sweeping.

Diagnosis. Funicle (Fig. 30) with $2 \mathrm{mps}$ on $\mathrm{fl}_{7}$ and $\mathrm{f}_{8}$ and $7 \mathrm{mps}$ on clava; mesoscutum (Fig. 33) without median longitudinal groove; frenum mostly smooth, 


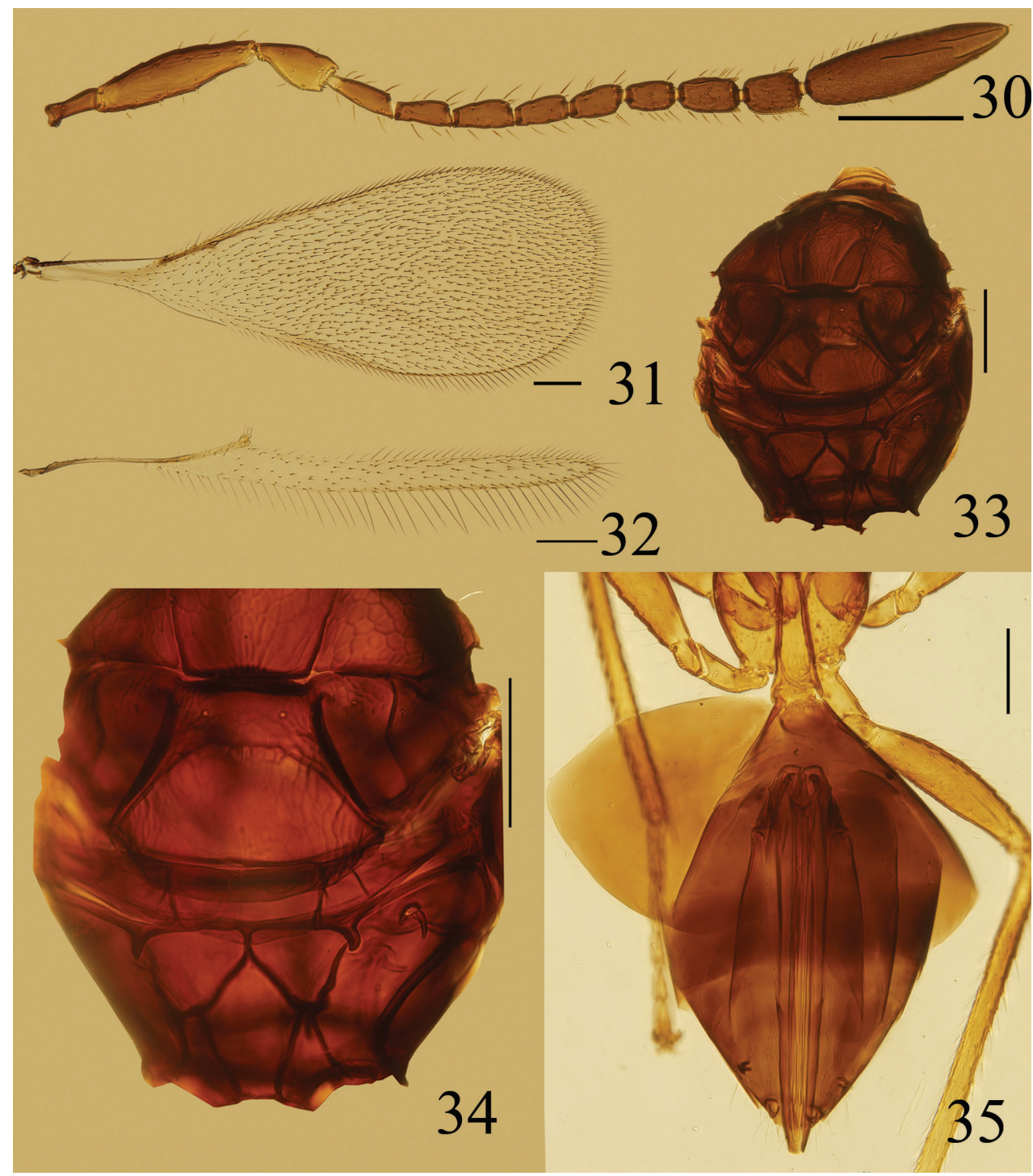

Figures 30-35. Ooctonus vulgatus, female (Laoyeling): $\mathbf{3 0}$ antenna $\mathbf{3}$ I fore wing $\mathbf{3 2}$ hind wing $\mathbf{3} \mathbf{3}$ mesosoma, dorsal 34 posterior part of mesoscutum to propodeum, dorsal 35 gaster. Scale bars $=100 \mu \mathrm{m}$.

except for obscure sculpture at lateral borders and sometimes also at anterior margin; propodeum (Fig. 34) with median areole abutting metascutellum; the median carina absent and replaced by the two carinae forming inner margin of dorsolateral areoles; plica almost straight and not divided anterodorsally, ending just anterior and medial to stub. 


\section{Acknowledgements}

This project was supported by the National Natural Science Foundation of China (Grant No. 31470652), by Research Fund for the Doctoral Program of Higher Education of China (Grant No. 201300621 10009). We are grateful to J. T. Huber, Canadian Forest Service, Ottawa, Canada, for providing reference of J.T. Huber (2012), and D. Chesters, Institute of Zoology, Chinese Academy of Sciences, Beijing, P. R. China, for correcting the English.

\section{References}

Gibson GAP (1997) Morphology and terminology. In: Gibson GAP, Huber JT, Woolley JB (Eds) Annotated keys to the Genera of Nearctic Chalcidoidea (Hymenoptera). NRC Research Press, Ottawa, 16-44. doi: 10.4039/entm120141fv

Huber JT (1988) The species groups of Gonatocerus Nees in North America with a revision of the sulphuripes and ater groups (Hymenoptera: Mymaridae). Memoirs of the Entomological Society of Canada 141: 1-109.

Huber JT (2012) Revision of Ooctonus (Hymenoptera: Mymaridae) in the Nearctic region. Journal of the Entomological Society of Ontario 143: 15-105.

Huber JT (2013) Revision of Ooctonus in the Neotropical region and comparison with Boudiennyia (Hymenoptera: Mymaridae). Zootaxa 3701(1):1-23. doi: 10.11646/zootaxa.3701.1.1

Huber JT, Read JD, van Noort S (2010) The genus Ooctonus Haliday (Hymenoptera: Mymaridae) in the Afrotropical region, with comments on other southern hemisphere species. African Entomology 18(2): 221-234. doi: 10.4001/003.018.0205

Noyes JS (1982) Collecting and preserving chalcid wasps (Hymenoptera: Chalcidoidea). Journal of Natural History 16: 315-334. doi: 10.1080/00222938200770261

Perkins RCL (1905) Leaf hoppers and their natural enemies (Pt. VI. Mymaridae, Platygasteridae). Bulletin of the Hawaiian Sugar Planters' Association Experiment Station (Entomology Series) 1(6): 187-203.

Triapitsyn SV (2010) Revision of the Palaearctic species and review of the Oriental species of Ooctonus (Hymenoptera: Mymaridae), with notes on extralimital taxa. Zootaxa 2381: 1-74. 